\title{
Validation of sepsis-induced coagulopathy score in critically ill patients with septic shock: post hoc analysis of a nationwide multicenter observational study in Japan
}

\author{
Chie Tanaka ${ }^{1} \cdot$ Takashi Tagami $^{2,3}$ (D) $\cdot$ Saori Kudo ${ }^{1} \cdot$ Akiko Takehara $^{1} \cdot$ Reo Fukuda $^{1} \cdot$ Fumihiko Nakayama $^{1}$. \\ Junya Kaneko ${ }^{1}$ - Yoshito Ishiki ${ }^{1}$. Shin Sato ${ }^{1}$. Masamune Kuno ${ }^{1} \cdot$ Kyoko Unemoto ${ }^{1}$
}

Received: 2 December 2020 / Revised: 7 April 2021 / Accepted: 8 April 2021 / Published online: 24 April 2021

(c) Japanese Society of Hematology 2021

\begin{abstract}
Coagulation disorder is a major cause of death in sepsis patients. Recently, sepsis-induced coagulopathy (SIC) scoring was developed as a new criterion for coagulopathy-associated sepsis. We aimed to evaluate the accuracy of the SIC score for predicting the prognosis of septic shock. We analyzed data from a multicenter observational study conducted from 2011 to 2013. We grouped the participants into those who did and did not use vasopressors, and compared the in-hospital mortality rates of SIC and non-SIC patients. Patients who needed vasopressors were considered to have septic shock. We performed survival analysis adjusted by factors independently associated with mortality. SIC developed in $66.4 \%$ of patients who used vasopressors and $42.2 \%$ of patients who did not. The in-hospital mortality difference between the SIC and non-SIC groups was statistically significant in those who needed vasopressors $(35.8 \%$ vs $27.9 \%, p<0.01)$. Cox regression analysis indicated that SIC was significantly correlated with mortality risk in patients who used vasopressors (hazard ratio [HR] 1.39; $95 \%$ confidence interval [CI] 1.13-1.70; $p<0.01$ ), but not in those who did not (HR 1.38; 95\% CI 0.81-2.34; $p=0.23$ ). In conclusion, the SIC score might be a good diagnostic indicator of fatal coagulopathy among sepsis patients who need vasopressors.
\end{abstract}

Keywords Sepsis $\cdot$ Coagulopathy $\cdot$ Sepsis-induced coagulopathy score $\cdot$ Septic shock

\begin{tabular}{|c|c|c|c|}
\hline \multicolumn{2}{|c|}{ Abbreviations } & IQR & Inter quartile range \\
\hline \multicolumn{2}{|r|}{ Acute Physiology and Chronic } & ISTH & $\begin{array}{l}\text { International Society on Thrombo- } \\
\text { sis and Hemostasis }\end{array}$ \\
\hline \multirow{3}{*}{$\begin{array}{l}\mathrm{CI} \\
\mathrm{DI}\end{array}$} & Confidence interval & JAAM & Japanese Association for Acute \\
\hline & Disseminated intravascular & & Medicine \\
\hline & coagulation & JSEPTIC-DIC study & The Japan Septic Disseminated \\
\hline \multirow{2}{*}{$\begin{array}{l}\mathrm{FL} \\
\mathrm{Hl}\end{array}$} & Fibrinogen degradation products & & Intravascular Coagulation study \\
\hline & Hazard ratio & LOS & The length of hospital stay \\
\hline ICL & Intensive care units & PT-INR & $\begin{array}{l}\text { Prothrombin time international } \\
\text { normalized ratio }\end{array}$ \\
\hline & $\begin{array}{l}\text { Takashi Tagami } \\
\text { t-tagami@nms.ac.jp }\end{array}$ & RRT & Renal replacement therapy \\
\hline & & SIC & Sepsis-induced coagulopathy \\
\hline \multirow{2}{*}{\multicolumn{2}{|c|}{$\begin{array}{l}\text { Department of Emergency and Critical Care Medicine, } \\
\text { Nippon Medical School Tama Nagayama Hospital, } \\
\text { Tama-shi, Tokyo 206-8512, Japan }\end{array}$}} & SIRS & $\begin{array}{l}\text { Systemic inflammatory response } \\
\text { syndrome }\end{array}$ \\
\hline & & SOFA & Sequential organ failure assessment \\
\hline
\end{tabular}

2 Department of Emergency and Critical Care Medicine, Nippon Medical School Musashikosugi Hospital, 1-396 Kosugimachi, Nakahara-ku, Kawasaki, Kanagawa 211-8533, Japan

3 Department of Clinical Epidemiology and Health Economics, School of Public Health, The University of Tokyo, Bunkyo, Tokyo 113-8654, Japan 


\section{Introduction}

Coagulopathy is one of the major factors associated with multiple organ dysfunction and death in patients with sepsis $[1,2]$. Coagulation abnormalities range from a slight decrease in platelet count to fatal disorders, such as disseminated intravascular coagulation (DIC). Coagulopathy in sepsis patients is caused by a compound relationship between the immune, inflammatory, and coagulation systems, and characterized by activated coagulation, disrupted anticoagulant systems, and excessive inhibition of fibrinolysis. The combined effect of these processes causes coagulation disorders to deteriorate to sepsis-associated DIC [3-5].

There are several diagnostic criteria for DIC, including the International Society on Thrombosis and Hemostasis (ISTH) non-overt DIC and Japanese Association for Acute Medicine (JAAM) DIC scores [6-8]. Previous studies have demonstrated that patients who had sepsis and were diagnosed with DIC in accordance with these two criteria presented high mortality rates [7-11]. In contrast, some papers have reported that the JAAM and ISTH DIC scores were not independent predictors for mortality among patients with sepsis $[12,13]$. These criteria were designed for patients with various conditions, such as infection, trauma, malignancy, or vascular abnormalities, and were not specialized for use in patients with sepsis.

Recently, the sepsis-induced coagulopathy (SIC) score was developed for evaluation of coagulation disorder in patients with sepsis [14]. The SIC score adopted the Sequential Organ Failure Assessment (SOFA) scoring system as a screening tool to evaluate organ dysfunction, according to the new definition of sepsis revised in 2016 (Sepsis-3 definition) [15]. This SIC is defined by the routine coagulation tests such as platelet count and prothrombin time ratio together with SOFA score [14]. The acute severe hypoxia of non-cardiogenic cause in critically ill patients is defined and classified as the acute respiratory distress syndrome (ARDS) using the Berlin definition [16]. Similarly, if the serious coagulation disorder regarding sepsis could be defined using the SIC score, it would be helpful for recognition of a fatal coagulopathy status and for deciding whether to provide anticoagulant therapy. Several previous studies have suggested that the SIC score might be a good predictive marker of mortality in patients with both sepsis and coagulopathy $[9,14,17-19]$. However, there were few reports assessed the relation between the SIC score and the most severe subset in sepsis, the so-called septic shock, which needs intensive care [20].

The aim of the present study was to evaluate the SIC score accuracy as a prognostic marker for patients with sepsis and septic shock, using a large nationwide cohort data.

\section{Materials and methods}

\section{Study design and setting of the study}

The ethics committee of the Nippon Medical School Tama Nagayama Hospital approved the present study. The current study followed the principle of the Declaration of Helsinki and was approved by the institutional review board of each participating hospital. The need to obtain written informed consent was waived because the participants, from whom the analyzed data were obtained, were anonymous.

This was a retrospective cohort study analyzing data from the Japan Septic Disseminated Intravascular Coagulation (JSEPTIC-DIC) study [21]. The study investigated the characteristics of patients with sepsis, who were admitted to the intensive care unit (ICU), and mainly focused on coagulation abnormalities and their treatments [21]. This dataset was previously published and was available to investigators for further study [21]. The JSEPTIC-DIC study was conducted in 42 ICUs of 40 institutions. This study's dataset excluded patients who were younger than 16 years or those who developed sepsis after ICU admission. Sepsis and septic shock were defined based on the International Sepsis Definitions Conference Criteria (Sepsis-2) published in 2003 [2]. The information in the dataset included age, sex, body weight, Acute Physiology and Chronic Health Evaluation (APACHE) II score [22]' SOFA score [23]' systemic inflammatory response syndrome (SIRS) score [24], primary infection site, blood culture results, daily results from laboratory tests, performed treatments, and outcomes.

\section{Participants and data collection}

The JSEPTIC-DIC dataset contained the data of patients treated from January 2011 to December 2013. Only adult patients admitted to the ICU, who were diagnosed with sepsis or septic shock based on Sepsis-2 guidelines, were included in this study. Cases with missing variables to calculate the coagulation abnormality scores, as shown in Table 1, were excluded.

We also excluded the patients who underwent warfarin/ acetylsalicylic acid/thrombolytic therapy before participation, and had a history of fulminant hepatitis, decompensated liver cirrhosis, other serious liver disorder, hematologic malignant disease, and treatment with any chemotherapy. These pre-existing hemostatic disorders possibly have an influence on the coagulation functions regardless of sepsis status at the study entry. The examined variables were as follows: age, sex, body weight, information regarding the past medical histories, information regarding any infection, platelet count, fibrinogen degradation products (FDP), D-dimer and fibrinogen levels, prothrombin time-international 
Table 1 Comparison of coagulopathy diagnostic criteria

\begin{tabular}{|c|c|c|c|c|}
\hline & Coagulation SOFA & ISTH overt DIC & JAAM & SIC \\
\hline $\begin{array}{l}\text { Underlying disease and Clini- } \\
\text { cal symptoms }\end{array}$ & & & $\begin{array}{l}\text { SIRS score } \\
0 \text { to } 2,0 \mathrm{p} \\
\geq 3,1 \mathrm{p}\end{array}$ & $\begin{array}{l}\text { SOFA four items } \\
0,0 \mathrm{p} \\
1,1 \mathrm{p} \\
\geq 2,2 \mathrm{p}\end{array}$ \\
\hline Platelet count $\left(10^{9} / 1\right)$ & $\begin{array}{l}\geq 150,0 \mathrm{p} \\
<150,1 \mathrm{p} \\
<100,3 \mathrm{p} \\
<50,3 \mathrm{p} \\
<20,4 \mathrm{p}\end{array}$ & $\begin{array}{l}>100,0 \mathrm{p} \\
<100,1 \mathrm{p} \\
<50,2 \mathrm{p}\end{array}$ & $\begin{array}{l}\geq 120,0 \mathrm{p} \\
\geq 80,<120,1 \mathrm{p} \\
<80,3 \mathrm{p}\end{array}$ & $\begin{array}{l}\geq 150,0 \mathrm{p} \\
<150,1 \mathrm{p} \\
<100,2 \mathrm{p}\end{array}$ \\
\hline Fibrinogen-related marker & & $\begin{array}{l}\text { Fibrin/fibrinogen degradation } \\
\text { products or } \mathrm{D} \text { dimer } \\
\text { No increase, } 0 \mathrm{p} \\
\text { Moderate increase, } 2 \mathrm{p} \\
\text { Strong increase, } 3 \mathrm{p}\end{array}$ & $\begin{array}{l}\text { Fibrin/fibrinogen degra- } \\
\text { dation products } \\
<10 \mathrm{mg} / \mathrm{l}, 0 \mathrm{p} \\
\geq 10,<25 \mathrm{mg} / \mathrm{l}, 1 \mathrm{p} \\
\geq 25 \mathrm{mg} / \mathrm{l}, 3 \mathrm{p}\end{array}$ & \\
\hline Fibrinogen & & $\begin{array}{l}>1.0 \mathrm{gL}^{-1} 0 \mathrm{p} \\
<1.0 \mathrm{gL}^{-1} 1 \mathrm{p}\end{array}$ & & \\
\hline Prothrombin time & & $\begin{array}{l}\text { Prolonged prothrombin time } \\
<3 \mathrm{~s}, 0 \mathrm{p} \\
3 \mathrm{~s}<,<6 \mathrm{~s}, 1 \mathrm{p} \\
\geq 6 \mathrm{~s}, 2 \mathrm{p}\end{array}$ & $\begin{array}{l}\text { PT-ratio } \\
<1.2,0 \mathrm{p} \\
\geq 1.2,1 \mathrm{p}\end{array}$ & $\begin{array}{l}\text { PT-INR } \\
\leq 1.2,0 \mathrm{p} \\
>1.2,1 \mathrm{p} \\
>1.4,2 \mathrm{p}\end{array}$ \\
\hline Diagnosis of DIC or SIC & & $\geq 5 \mathrm{p}$ & $\geq 4 \mathrm{p}$ & $\begin{array}{l}\geq 4 p \text { (with PT-INR plus } \\
\text { Platelet count exceed- } \\
\text { ing 2) }\end{array}$ \\
\hline
\end{tabular}

SOFA four items is the sum the four items (respiratory SOFA, cardiovascular SOFA, hepatic SOFA, and renal SOFA)

Adapted from Ding R, Blood coagulation and fibrinolysis: an international journal in haematosis and thrombosis. 2018; 29 (6):551-8

SIRS systemic inflammatory response syndrome, SOFA sequential organ failure assessment ISTH International Society on Thrombosis and Hemostasis, JAAM Japanese Association for Acute Medicine, SIC sepsis-induced coagulopathy, DIC disseminated intravascular coagulation

normalized ratio (PT-INR), SIRS score, respiratory SOFA score, cardiovascular SOFA score, hepatic SOFA score, renal SOFA score, information concerning the performed critical care treatments (such as ventilator and vasopressor days) and the performed treatments for coagulopathy (i.e., heparinoids and thrombomodulin), and the length of hospital stay (LOS). Numerical values of vital signs were not contained.

\section{Outcome measures}

The primary outcome measure was the in-hospital mortality rates. The secondary outcome measures were the ICU-, ventilator, and renal replacement therapy (RRT)-free days, the LOS from ICU admission, the length of ICU stay, and the mortality rates in the ICU.

\section{Definition}

We used three coagulopathic disorder scores (SIC, JAAM DIC, and ISTH DIC scores) to assess the coagulopathy disorder secondary to sepsis. We computed these scores from five variables (platelet count, fibrinogen-related marker, fibrinogen, prothrombin time, and clinical symptoms) according to their criteria shown in Table $1[6-8,14]$. We checked the cumulative scores and determined whether the patient was diagnosed as having SIC, JAAM DIC, and ISTH DIC, according to the criteria.

As the JSEPTIC-DIC dataset did not contain information of vital signs and the amount of infusion and vasopressor agents, we could not precisely discriminate septic shock patients according to Sepsis-3 definition. Therefore, in this study, we regarded the patients who underwent vasopressor to have septic shock.

We used the ICU-, ventilator-, RRT-, and vasopressorfree days as secondary outcomes in the current study. In particular, we calculated the ventilator-free days as follows $[25,26]$ :

1) Ventilator-free days =0: when the patient died within 28 days of mechanical ventilation;

2) Ventilator-free days $=28-x$ : when mechanical ventilation was successfully discontinued within 28 days ( $x$ corresponded to the number of days spent receiving mechanical ventilation);

3) Ventilator-free days =0: when the patient underwent mechanical ventilation for $\geq 28$ days).

We calculated other failure-free days using the same method. 


\section{Statistical analysis}

First, the following variables were calculated: SIC score, JAAM DIC score, ISTH DIC score, ICU-free days, ventilator-free days, and RRT-free days. Next, we grouped all patients into those with and without vasopressor use. Third, comparisons of patients' characteristics and covariates between those with and without SIC were conducted in the vasopressor group. The analyses of continuous variables were performed using Student's $t$-test or Mann-Whitney $U$ test, and the comparison of categorical variables was performed using the $\chi^{2}$ or Fisher's test. Thereafter, we compared the patients' background and covariates between those with and without SIC in the no vasopressor group. Finally, we performed survival analysis using Cox proportional hazard regression analysis and selected the covariates that were independently associated with mortality, in accordance with previous studies [27]. The examined factors were age, sex, SIC, and treatment for DIC (antithrombin, thrombomodulin, protease inhibitors, and heparinoids) [9, 10, 12, 28, 29].

The numerical values in the text and the tables represent the medians and the inter quartile ranges; in contrast, the ICU-, ventilator-, RRT-, and vasopressor-free days were presented as means and standard deviations (SDs) [26]. All hypotheses were two-sided, and values of $p<0.05$ were considered statistically significant. All analyses were performed using SPSS software version 23 (IBM Corp., Armork, NY, USA).

\section{Results}

This study included 1894 patients (1453 in the vasopressor group and 441 in the no vasopressor group [Fig. 1]). Table 2 shows the trend of patients' demographics and clinical characteristics of the vasopressor and no vasopressor groups. In the vasopressor and no vasopressor groups, 965 (66.4\%)

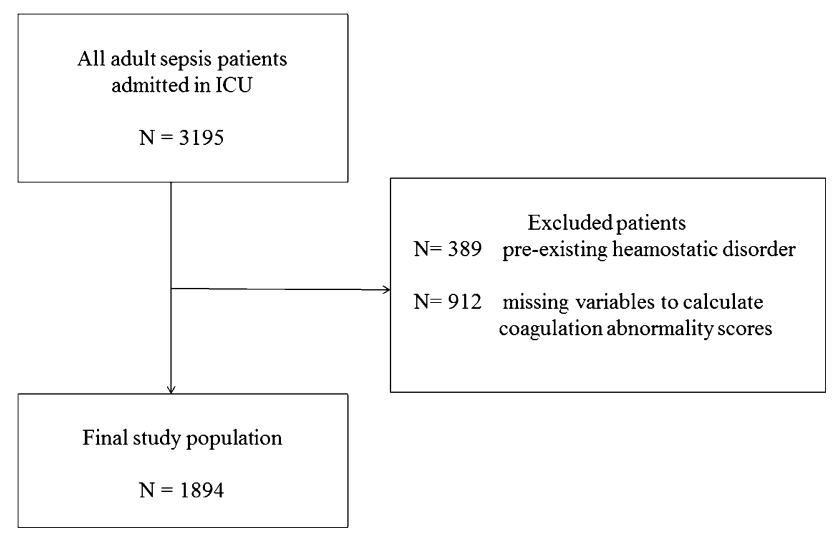

Fig. 1 Patient selection and $186(42.2 \%)$ patients were diagnosed with SIC. As for patients who needed vasopressor, some variables, including the APACHE II score, lactate levels, platelet count, FDP, fibrinogen levels, antithrombin levels, and PT-INR, significantly differed between those with and without SIC. There were significant differences regarding the total SOFA score, the diagnosis of ISTH DIC, the diagnosis of JAAM DIC, and the coagulation SOFA score between those with and without SIC in both groups.

Regarding the primary outcome, the difference of the inhospital mortality between the patients with and without SIC was statistically significant in the vasopressor group; however, the difference in the no vasopressor group was not significant ( $35.8 \%$ vs $27.9 \%, p<0.01 ; 15.6 \%$ vs $12.2 \%$, $p=0.33$, respectively) Table 3 . Regarding the secondary outcomes, in the vasopressor group, there was a significant difference in the mortality rates in the ICU between the two groups $(24.0 \%$ vs $16.4 \%, p<0.01)$. Moreover, in the vasopressor group, the RRT-free days and ICU-free days were significantly lower in those with SIC than in those without SIC (mean days \pm SD: $17.6 \pm 12.3$ vs $20.3 \pm 11.7$, $p<0.01,12.0 \pm 10.2$ vs $13.2 \pm 10.1, p=0.03$, respectively). However, in the no vasopressor group, the RRT- and ICUfree days were not significantly different between those with SIC and without SIC (mean days \pm SD: $23.0 \pm 10.0$ vs $24.2 \pm 9.1, p=0.22 ; 16.9 \pm 9.7$ vs $17.8 \pm 8.6, p=0.29$, respectively).

As shown in Fig. 2, the Cox regression analysis revealed that the SIC diagnosis was significantly associated with the risk of in-hospital mortality for sepsis patients who needed vasopressor adjusted for factors independently associated with mortality (Hazard ratio [HR] 1.39; 95\% confidence interval $[\mathrm{CI}] 1.13-1.70 ; p<0.01)$. However, the SIC diagnosis had no relation with the risk of mortality for sepsis patients without vasopressor (HR 1.38; 95\% CI 0.81-2.34; $p=0.23$ ).

\section{Discussion}

The current study results, based on the data from a multicenter observational database in Japan, suggested that the SIC score confer a good prognostic value to recognize lethal coagulation abnormality among sepsis patients who need vasopressor.

This study investigated the data of 1894 patients with sepsis who were hospitalized in 42 ICUs. The novelty of this study was the inclusion of a large cohort of sepsis patients with coagulation abnormality noticing blood pressure. Several previous studies have demonstrated that the SIC diagnosis might be a good predictor of mortality among patients with sepsis and coagulopathy [9, 14, 17-19]. The current study showed that the SIC score was associated 
Table 2 Demographics and clinical characteristics of study patients

\begin{tabular}{|c|c|c|c|c|c|c|}
\hline \multirow[t]{2}{*}{ Variables } & \multicolumn{3}{|c|}{ Vasopressor (1453) } & \multicolumn{3}{|c|}{ No vasopressor (441) } \\
\hline & $\operatorname{SIC}(n=965)$ & Non-SIC $(n=488)$ & $p$ & $\operatorname{SIC}(n=186)$ & Non-SIC $(n=255)$ & $p$ \\
\hline Age, years & $71(61-80)$ & $73(64-80)$ & 0.01 & $71(60-80)$ & $71(60-80)$ & 0.60 \\
\hline Male & $564(58.4 \%)$ & $292(59.8 \%)$ & 0.65 & $118(63.4 \%)$ & $145(56.9 \%)$ & 0.17 \\
\hline Body weight, $\mathrm{kg}$ & $55(47-65)$ & $54(46-64)$ & 0.15 & $58(48-66)$ & $56(47-65)$ & 0.07 \\
\hline APACHE II & $24(18-30)$ & $22(17-27)$ & $<0.01$ & $19(13-22)$ & $18(13-23)$ & 0.67 \\
\hline Lactate, $\mathrm{mmol} / \mathrm{L}$ & $3.7(2.1-6.8)$ & $2.9(1.7-5.0)$ & $<0.01$ & $2.3(1.4-3.7)$ & $1.8(1.1-3.3)$ & 0.02 \\
\hline White blood cell, $10^{3} / \mu \mathrm{L}$ & $10.3(3.7-17.8)$ & $12.0(6.7-18.6)$ & 0.19 & $14.3(8.4-20.9)$ & $12.9(8.6-19.0)$ & 0.07 \\
\hline Platelet, $10^{3} / \mu \mathrm{L}$ & $82(50-126)$ & $199(158-266)$ & $<0.01$ & $81(49-121)$ & $208(160-268)$ & $<0.01$ \\
\hline FDP, $\mu \mathrm{g} / \mathrm{mL}$ & $24.0(13.0-59.4)$ & $16.0(9.1-27.1)$ & $<0.01$ & $20.7(11.0-44.8)$ & $13.3(7.4-43.5)$ & 0.03 \\
\hline Hemoglobin, g/dL & $10.4(8.8-12.1)$ & $11.3(9.6-13.1)$ & $<0.01$ & $11.4(9.8-13.0)$ & $11.3(9.8-13.1)$ & 0.89 \\
\hline Fibrinogen, mg/Dl & $347(222-498)$ & 435 (318-597) & $<0.01$ & $454(341-655)$ & $500(362-659)$ & 0.33 \\
\hline Antithrombin, $\%$ & $51(40-62)$ & $67(54-79)$ & $<0.01$ & $55(49-68)$ & $75(60-86)$ & $<0.01$ \\
\hline PT-INR & $1.5(1.3-1.8)$ & $1.2(1.1-1.3)$ & $<0.01$ & $1.4(1.2-1.6)$ & $1.1(1.0-1.2)$ & $<0.01$ \\
\hline Total SOFA & $11(9-14)$ & $8(6-11)$ & $<0.01$ & $7(5-9)$ & $5(3-7)$ & $<0.01$ \\
\hline ISTH DIC (+) & $470(48.7 \%)$ & $0(0 \%)$ & $<0.01$ & $68(36.6 \%)$ & $2(0.8 \%)$ & $<0.01$ \\
\hline The median score of ISTH DIC & $4.0(4.0-5.0)$ & $2.0(1.0-3.0)$ & $<0.01$ & $4.0(3.0-5.0)$ & $2.0(0-3.0)$ & $<0.01$ \\
\hline JAAM DIC (+) & $717(74.3 \%)$ & $128(26.2 \%)$ & $<0.01$ & $136(73.1 \%)$ & $53(20.8 \%)$ & $<0.01$ \\
\hline The median score of JAAM DIC & $5.0(3.0-6.0)$ & $2.0(1.0-4.0)$ & $<0.01$ & $5.0(3.0-6.0)$ & $2.0(1.0-3.0)$ & $<0.01$ \\
\hline The median score of SIC & $5.0(4.0-6.0)$ & $3.0(2.0-3.0)$ & $<0.01$ & $4.0(4.0-5.0)$ & $2.0(2.0-3.0)$ & $<0.01$ \\
\hline Coagulation SOFA at day1 & $2.0(1.0-2.0)$ & $0(0)$ & $<0.01$ & $2.0(1-2.3)$ & $0(0)$ & $<0.01$ \\
\hline Primary infection site & & & $<0.01$ & & & $<0.01$ \\
\hline Unknown & $52(5.4 \%)$ & $15(3.1 \%)$ & & $17(9.1 \%)$ & $8(3.1 \%)$ & \\
\hline Catheter related & $10(1.0 \%)$ & $8(1.6 \%)$ & & $0(0 \%)$ & $2(0.8 \%)$ & \\
\hline Bone/soft tissue & $124(12.8 \%)$ & $50(10.2 \%)$ & & $28(15.1 \%)$ & $40(15.7 \%)$ & \\
\hline Cardiovascular & $25(2.6 \%)$ & $7(1.4 \%)$ & & $5(2.7 \%)$ & $4(1.6 \%)$ & \\
\hline Central nervous system & $23(2.4 \%)$ & $6(1.2 \%)$ & & $7(3.8 \%)$ & $13(5.1 \%)$ & \\
\hline Urinary tract & $169(17.5 \%)$ & $51(10.5 \%)$ & & $39(21.0 \%)$ & $51(20.0 \%)$ & \\
\hline Lung/thoracic & $171(17.7 \%)$ & $181(37.1 \%)$ & & $33(17.7 \%)$ & $87(34.1 \%)$ & \\
\hline Abdomen & $371(38.4 \%)$ & $160(32.8 \%)$ & & $54(29.0 \%)$ & $46(18.0 \%)$ & \\
\hline Other & $20(2.1 \%)$ & $10(2.0 \%)$ & & $3(1.6 \%)$ & $4(1.6 \%)$ & \\
\hline Treatment for DIC & $651(67.5 \%)$ & $204(41.8 \%)$ & $<0.01$ & $86(46.2 \%)$ & $56(22.0 \%)$ & $<0.01$ \\
\hline Antithrombin & $460(47.7 \%)$ & $121(24.8 \%)$ & $<0.01$ & $47(25.3 \%)$ & $31(12.2 \%)$ & $<0.01$ \\
\hline Thrombomodulin & $391(40.5 \%)$ & $115(23.6 \%)$ & $<0.01$ & $52(28.0 \%)$ & $20(7.8 \%)$ & $<0.01$ \\
\hline Protease inhibitors & $170(17.6 \%)$ & $45(9.2 \%)$ & $<0.01$ & $17(9.1 \%)$ & $22(8.6 \%)$ & 0.87 \\
\hline Heparinoids & $60(6.2 \%)$ & $24(4.9 \%)$ & 0.34 & $14(7.5 \%)$ & $10(3.9 \%)$ & 0.14 \\
\hline
\end{tabular}

Data were given as number of positive observations/ total number of observations (percentage) or as median (interquartile range)

SIC sepsis-induced coagulopathy, APACHE II Acute Physiology and Chronic Health Evaluation II, FDP fibrinogen degradation products, PTINR prothrombin time-international normalized ratio, SOFA sequential organ failure assessment, ISTH International Society on Thrombosis and Hemostasis, JAAM Japanese Association for Acute Medicine, DIC disseminated intravascular coagulation

with mortality in the vasopressor group and had no relation to mortality in the no vasopressor group. Especially, we implied that the SIC score might be a useful prognostic tool for septic shock that was the most severe subset in sepsis according to Sepsis-3 criteria, whereas the SIC score was possibly useless for patients without septic shock.

Previous works have elucidated the mechanism of coagulation disorders in sepsis [3-5]. Coagulation plays a key role in the innate immune system under certain circumstances: the causative agent and the mediators generated by the inflammatory response in sepsis, such as pathogen-associated molecular patterns, protease-activated responses, damage-associated molecular patterns, neutrophil extracellular traps, other tissue factors, thrombus formation, and the plasminogen activator inhibitor-1 that suppresses fibrinolysis [17, 30, 31]. Moreover, DIC secondary to infection is characterized by the systemic activation of coagulation and often leads to organ dysfunction complications caused by a 
Table 3 Measurement of primary and secondary outcomes using SIC score

\begin{tabular}{|c|c|c|c|c|c|c|}
\hline \multirow[t]{2}{*}{ Variables } & \multicolumn{3}{|c|}{ Vasopressor (1453) } & \multicolumn{3}{|c|}{ No vasopressor (441) } \\
\hline & $\operatorname{SIC}(n=965)$ & Non-SIC $(n=488)$ & $p$ & $\operatorname{SIC}(n=186)$ & Non-SIC $(n=255)$ & $p$ \\
\hline In-hospital mortality & $345(35.8 \%)$ & $136(27.9 \%)$ & $<0.01$ & $29(15.6 \%)$ & $31(12.2 \%)$ & 0.33 \\
\hline RRT-free days, mean days (SD) & $17.6(12.3)$ & $20.3(11.7)$ & $<0.01$ & $23.0(10.0)$ & $24.2(9.1)$ & 0.22 \\
\hline Ventilator-free days, mean days (SD) & $14.7(11.4)$ & $15.5(11.0)$ & 0.19 & $21.9(9.8)$ & $22.0(9.3)$ & 0.92 \\
\hline ICU-free days, mean days (SD) & $12.0(10.2)$ & $13.2(10.1)$ & 0.03 & $16.9(9.7)$ & $17.8(8.6)$ & 0.29 \\
\hline Length of ICU stay, median days (IQR) & $8.0(4.0-15.0)$ & $8.0(5.0-15.0)$ & 0.21 & $5.5(3.0-11.0)$ & $6.0(3.0-12.0)$ & 0.32 \\
\hline $\begin{array}{l}\text { Length of hospital stay from ICU admis- } \\
\text { sion, median days (IQR) }\end{array}$ & $28(13-56)$ & $27(15-53)$ & 0.99 & $25(14-45)$ & $22(11-45)$ & 0.74 \\
\hline Mortality in ICU & $232(24.0 \%)$ & $80(16.4 \%)$ & $<0.01$ & $13(7.0 \%)$ & $15(5.9 \%)$ & 0.69 \\
\hline
\end{tabular}

$S I C$ sepsis-induced coagulopathy, $R R T$ renal replacement therapy, $I C U$ intensive care unit, IQR interquartile range, $S D$ standard deviation

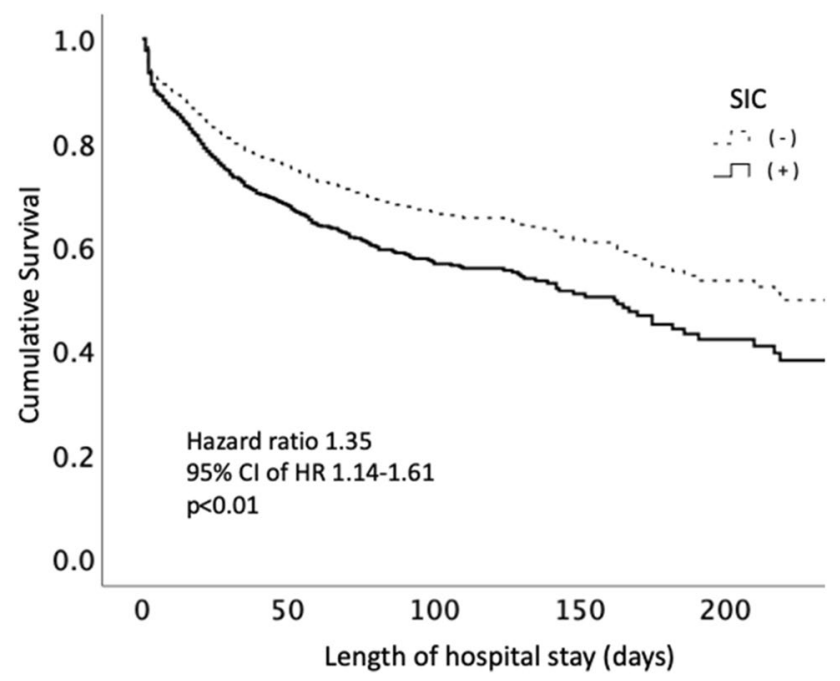

Fig. 2 Survival analysis using cox regression among sepsis patients who needed vasopressor

microcirculatory disorder [3, 17]. In the vasopressor group, the APACHE II score, lactate levels, and the total SOFA score were significantly higher in those with SIC than in those without SIC. Moreover, the in-hospital mortality was lower in those with SIC than in those without SIC. Consequently, we speculated that the patients who suffered from a more severe general condition and septic shock had enhanced inflammation, and they might have developed lethal coagulation complications more frequently.

Another aim of the current study was to examine the use of the SIC score to assess coagulation abnormality associated with sepsis. In general, the DIC, JAAM DIC, and ISTH overt-DIC scores, and the Japanese Ministry of Health and welfare DIC criteria, were defined as systemic intravascular activation of coagulation despite differences in the underlying diseases [32]. In contrast, the SIC score focused only on coagulation disorder of sepsis patients. The SIC scoring system has two advantages: it is simple and easy to use, and it is suitable to assess the mechanism of sepsis-associated DIC. The SIC score was designed to fit to Sepsis-3 criteria, and it consisted of only three variables: the platelet count, PT-INR, and the four SOFA scores [14]. In Sepsis-3, the SOFA score plays an important role to identify sepsis. Therefore, the SIC score was regarded as a simpler and easier method to assess coagulation disorder than other DIC criteria. In addition, sepsis associated DIC is featured by thrombocytopenia, elevated levels of fibrin-related markers, and the coagulation activation with over suppression of fibrinolysis $[4,14$, $30,32]$. The fibrinogen level is not a good marker for the thrombotic phenotype of DIC that is associated with the infection. The SIC score excluded the fibrinogen levels in the scoring while the ISTH overt-DIC score did not, thus, increasing the efficacy of the SIC score in sepsis patients.

The data of ICU patients were analyzed and, therefore, both in-hospital mortality and intensive care procedures (RRT and ventilator management) were assessed. As shown in Table 3, in the vasopressor group, the diagnosis of SIC showed higher in-hospital and ICU mortality rates, lower RRT-free days, and lower ICU-free days than that of non-SIC. In contrast, there were no differences regarding these outcomes between those with and without SIC in the no vasopressor group. Furthermore, as the failurefree days were the composite outcome measures, e.g., the ventilator-free days consisted of mortality and ventilation duration [26], we performed survival analysis using a cox proportional hazard model to evaluate mortality adjusted by age, sex, the diagnosis of SIC, and treatment for DIC. In the vasopressor group, the survival rate was significantly higher for patients without SIC than for those with SIC Fig. 2. Moreover, in the no vasopressor group, there was no significant difference in the survival rate between patients with and without SIC. These results suggested that SIC on the admission to the ICU might be a good prognostic criterion of sepsis-associated coagulopathy, which 


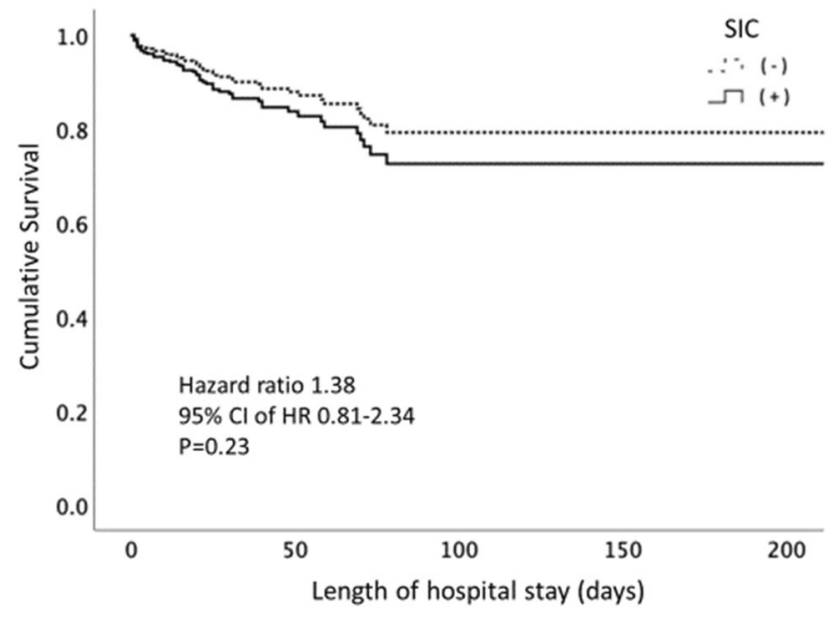

Fig. 3 Survival analysis using cox regression among sepsis patients without vasopressor

predicted high mortality and the high need for intensive care for sepsis patients with vasopressor (Fig. 3).

There were several limitations in the current study. First, this was a retrospective cohort study in which the participants with missing variables were excluded. Therefore, confounding factors might have affected the assessment of the prognostic accuracy of the SIC score. Second, the patients in this database were diagnosed using Sepsis-2 definition. As this dataset did not include information of the baseline SOFA score, it is difficult to diagnose sepsis according to Sepsis-3 definition. On the supposition that the baseline SOFA score was zero, $98.7 \%$ of the participants had two or more SOFA scores on admission to the ICU; thus, we supposed that we would extract similar results and outcomes among sepsis patients even when the diagnosis was made based on Sepsis- 3 criteria. Third, the analysis was performed without adjusting for some variables affecting mortality, such as the infection focus, causative pathogens, the needs for source control, antimicrobial treatments, and treatments for coagulation abnormality. Moreover, we could not obtain information regarding the cause of death from this dataset. Therefore, the direct effect of these factors on the SIC scoring could not be assessed.

The findings of our post hoc analysis, which assessed the condition of patients with sepsis in Japan, suggested that the diagnosis of SIC might be a new and simple criterion to recognize fatal sepsis-associated coagulopathy during ICU admission among most critically ill patients who need vasopressor to manage septic shock. Further large prospective studies are required to confirm our results.

\section{Declarations}

Conflict of interest The authors declare that they have no conflict of interests.

\section{References}

1. Zeerleder S, Hack CE, Wuillemin WA. Disseminated intravascular coagulation in sepsis. Chest. 2005;128:2864-75.

2. Levi M, de Jonge E, van der Poll T. Sepsis and disseminated intravascular coagulation. J Thromb Thrombolysis. 2003; 16:43-7.

3. Levi M, van der Poll T. Coagulation and sepsis. Thromb Res. 2017;149:38-44.

4. Iba T, Ogura H. Role of extracellular vesicles in the development of sepsis-induced coagulopathy. J Intensive Care. 2018;6:68.

5. Lipinska-Gediga M. Coagulopathy in sepsis-a new look at an old problem. Aenaesthesiol Intensive Ther. 2016;48:352-9.

6. Taylor FB Jr, Toh CH, Hoots WK, Wada H, Levi M. Towards definition, clinical and laboratory criteria, and a scoring system for disseminated intravascular coagulation. Thromb Haemost. 2001;86:1327-30.

7. Gando S, Saitoh D, Ogura H, Mayumi T, Koseki K, Ikeda T, et al. Natural history of disseminated intravascular coagulation diagnosed based on the newly established diagnostic criteria for critically ill patients: results of a multicenter, prospective survey. Crit Care Med. 2008;36:145-50.

8. Gando S, Iba T, Eguchi Y, Ohtomo Y, Okamoto K, Koseki K, et al. A multicenter, prospective validation of disseminated intravascular coagulation diagnostic criteria for critically ill patients: comparing current criteria*. Crit Care Med. 2006;34:625-31.

9. Ding R, Wang Z, Lin Y, Liu B, Zhang Z, Ma X. Comparison of a new criteria for sepsis-induced coagulopathy and International Society on Thrombosis and Haemostasis disseminated intravascular coagulation score in critically ill patients with sepsis 3.0: a retrospective study. Blood Coagul Fibrinolysis. 2018;29:551-8.

10. Cauchie P, Cauchie C, Boudjeltia KZ, Carlier E, Deschepper N, Govaerts D, et al. Diagnosis and prognosis of overt disseminated intravascular coagulation in a general hospital-meaning of the ISTH score system, fibrin monomers, and lipoprotein-C-reactive protein complex formation. Am J Hematol. 2006;81:414-9.

11. Gando S, Saitoh D, Ogura H, Fujishima S, Mayumi T, Araki T, et al. A multicenter, prospective validation study of the Japanese Association for Acute Medicine disseminated intravascular coagulation scoring system in patients with severe sepsis. Critical care (London, England). 2013;17:R111.

12. Saito S, Uchino S, Hayakawa M, Yamakawa K, Kudo D, Iizuka $\mathrm{Y}$, et al. Epidemiology of disseminated intravascular coagulation in sepsis and validation of scoring systems. J Crit Care. 2019;50:23-30.

13. Ha SO, Park SH, Hong SB, Jang S. Performance evaluation of five different Disseminated intravascular coagulation (DIC) diagnostic criteria for predicting mortality in patients with complicated sepsis. J Korean Med Sci. 2016;31:1838-45.

14. Iba T, Nisio MD, Levy JH, Kitamura N, Thachil J. New criteria for sepsis-induced coagulopathy (SIC) following the revised sepsis definition: a retrospective analysis of a nationwide survey. BMJ Open. 2017;7:e017046.

15. Singer M, Deutschman CS, Seymour CW, Shankar-Hari M, Annane D, Bauer M, et al. The third international consensus definitions for sepsis and septic shock (Sepsis-3). JAMA. 2016;315:801-10. 
16. Force ADT, Ranieri VM, Rubenfeld GD, Thompson BT, Ferguson $\mathrm{ND}$, Caldwell E, et al. Acute respiratory distress syndrome: the Berlin definition. JAMA. 2012;307:2526-33.

17. Iba T, Watanabe E, Umemura $Y$, Wada $T$, Hayashida K, Kushimoto $\mathrm{S}$, et al. Sepsis-associated disseminated intravascular coagulation and its differential diagnoses. J Intensive Care. 2019;7:32.

18. Iba T, Arakawa M, Di Nisio M, Gando S, Anan H, Sato K, et al. Newly proposed sepsis-induced coagulopathy precedes international society on thrombosis and haemostasis overt-disseminated intravascular coagulation and predicts high mortality. J Intensive Care Med. 2020;35:643-9.

19. Yamakawa K, Yoshimura J, Ito T, Hayakawa M, Hamasaki T, Fujimi S. External validation of the two newly proposed criteria for assessing coagulopathy in sepsis. Thromb Haemost. 2019;119:203-12.

20. Helms J, Severac F, Merdji H, Clere-Jehl R, Francois B, Mercier E, et al. Performances of disseminated intravascular coagulation scoring systems in septic shock patients. Ann Intensive Care. 2020;10:92.

21. Hayakawa M, Yamakawa K, Saito S, Uchino S, Kudo D, Iizuka $\mathrm{Y}$, et al. Nationwide registry of sepsis patients in Japan focused on disseminated intravascular coagulation 2011-2013. Sci Data. 2018;5:180243.

22. Knaus WA, Draper EA, Wagner DP, Zimmerman JE. APACHE II: a severity of disease classification system. Crit Care Med. 1985;13:818-29.

23. Vincent JL, de Mendonca A, Cantraine F, Moreno R, Suter PM, Sprung CL, et al. Use of the SOFA score to assess the incidence of organ dysfunction/failure in intensive care units: results of a multicenter, prospective study. Working group on "sepsis-related problems" of the European Society of Intensive Care Medicine. Crit Care Med. 1998;26:1793-800.

24. Bone RC, Balk RA, Cerra R, Dellinger RP, Fein AM, Knaus WA, Schein RMH, et al. Definitions for sepsis and organ failure and guidelines for the use of innovative therapies in sepsis. The ACCP/SCCM Consensus Conference Committee. American
College of Chest Physicians/Society of Critical Care Medicine. Chest. 1992;101:1644-55.

25. Schoenfeld DA, Bernard GR. Statistical evaluation of ventilator-free days as an efficacy measure in clinical trials of treatments for acute respiratory distress syndrome. Crit Care Med. 2002;30:1722-7.

26. Yehya N, Harhay MO, Curley MAQ, Schoenfeld DA, Reeder RW. Reappraisal of ventilator-free days in critical care research. Am J Respir Crit Care Med. 2019;200:828-36.

27. Sun G-W, Shook TL, Kay GL. Inappropriate use of bivariable analysis to screen risk factors for use in multivariable analysis. J Clin Epidemiol. 1996;49:907-16.

28. Iba T, Arakawa M, Levy JH, Yamakawa K, Koami H, Hifumi $\mathrm{T}$, et al. Sepsis-induced coagulopathy and Japanese association for acute medicine DIC in coagulopathic patients with decreased antithrombin and treated by antithrombin. Clin Appl Thromb Hemost. 2018;24:1020-6.

29. Yatabe T, Inoue S, Sakamoto S, Sumi Y, Nishida O, Hayashida K, et al. The anticoagulant treatment for sepsis induced disseminated intravascular coagulation; network meta-analysis. Thromb Res. 2018; 171:136-42.

30. Semeraro N, Ammollo CT, Semeraro F, Colucci M. Coagulopathy of acute sepsis. Semin Thromb Hemost. 2015;41:650-8.

31. Engelmann B, Massberg S. Thrombosis as an intravascular effector of innate immunity. Nat Rev Immunol. 2013;13:34-45.

32. Iba T, Umemura Y, Watanabe E, Wada T, Hayashida K, Kushimoto S, et al. Diagnosis of sepsis-induced disseminated intravascular coagulation and coagulopathy. Acute Med Surg. 2019;6:223-32.

Publisher's Note Springer Nature remains neutral with regard to jurisdictional claims in published maps and institutional affiliations. 